\title{
Duality formulas of the Special Values of Multiple Polylogarithms
}

\author{
OKUDA Jun-ichi
}

\begin{abstract}
The special values of multiple polylogarithms, which include multiple zeta values, appear in several fields of mathematics and physics. Many kinds of their linear relations are investigated as well as their algebraic relations. From the viewpoint of a connection matrix of Fuchsian equations, two kinds of duality of these values are derived.
\end{abstract}

\section{Introduction}

In 2] the multiple polylogarithm is defined by

$$
\lambda\left(\begin{array}{lll}
s_{1} & \ldots & s_{k} \\
b_{1} & \ldots & b_{k}
\end{array}\right)=\sum_{m_{1}=k}^{\infty} \sum_{m_{1}>m_{2}>\cdots>m_{k}>0} \frac{b_{1}^{-\left(m_{1}-m_{2}\right)} \cdots b_{k-1}^{-\left(m_{k-1}-m_{k}\right)} b_{k}^{-m_{k}}}{m_{1}^{s_{1}} \cdots m_{k-1}^{s_{k-1}} m_{k}^{s_{k}}}
$$

and many relations, reductions, and explicit evaluations of special values of them are investigated. In [1] a class of the special values of the multiple polylogarithm are called multiple $L$-values. For any $m \in \mathbb{Z}_{>0}$, we put $R_{m}=$ $\mathbb{Z} / m \mathbb{Z}$ and $\zeta_{m}=e^{2 \pi \sqrt{-1} / m}$. The multiple $L$-value of positive integers $k_{i}$ and $a_{i} \in R_{m}(i=1, \ldots, r)$ is defined by

$$
L\left(k_{1}, \ldots, k_{r} ; a_{1}, \ldots, a_{r}\right)=\lambda\left(\begin{array}{cccc}
k_{1} & \ldots & k_{r-1} & k_{r} \\
\zeta_{m}^{-a_{1}} & \ldots & \zeta_{m}^{-a_{r-1}} & \zeta_{m}^{-a_{r}}
\end{array}\right)
$$

In particular the case $m=1$ is known as multiple zeta values

$$
\zeta\left(k_{1}, k_{2}, \ldots, k_{r}\right)=\sum_{m_{1}>m_{2}>\cdots>m_{r}>0} \frac{1}{m_{1}^{k_{2}} m_{2}^{k_{2}} \cdots m_{r}^{k_{r}}},
$$

and many kinds of relations and properties are discovered. Also the case $m=6$ is discussed in [3] from the point of view of quantum field theory and 
some relations are obtained. The main purpose of the study of these values is to discover all the linear relations explicitly.

In 4 the $\mathrm{KZ}$ associator $\Phi(X, Y)$ is defined by

$$
\Phi(X, Y)=G_{1}^{-1}(z) G_{0}(z)
$$

where $G_{0}$ and $G_{1}$ are the solutions of the Fuchsian equation called the formal $\mathrm{KZ}$ equation

$$
\frac{d G}{d z}=\left(\frac{X}{z}+\frac{Y}{z-1}\right) G, \quad G(z) \in \mathbb{C}\langle\langle X, Y\rangle\rangle,
$$

characterized by the following asymptotic properties respectively:

$$
G_{0}(z) \times z^{-X} \rightarrow 1 \quad(z \rightarrow 0) \quad \text { and } \quad G_{1}(z) \times(1-z)^{-Y} \rightarrow 1 \quad(z \rightarrow 1) .
$$

Here $z^{-X}=\exp \left(\sum_{n=0}^{\infty}(-X \log z)^{n} / n !\right)$ and so on. It is conjectured that all the relations for multiple zeta values can be deduced from the relations which the $\mathrm{KZ}$ associator satisfies. So the $\mathrm{KZ}$ associator is a very important generating function of multiple zeta values. For example, the coefficients of the relation

$$
\Phi(X, Y)^{-1}=\Phi(Y, X)
$$

give rise to the duality formula for multiple zeta values. From this point of view some studies have been developed (c.f. [6, 5, 9]).

In this paper we consider the next generalization of the $\mathrm{KZ}$ associator. Using the connection matrices of specified solutions, we investigate the relations among the special values of multiple polylogarithms including multiple $L$-values. For any finite subset $\Sigma \subset \mathbb{C}$ we define the generalization of the formal $\mathrm{KZ}$ equation as follows:

$$
\frac{d H}{d z}=\left(\sum_{a \in \Sigma} \frac{X_{a}}{z-a}\right) H, \quad H(z) \in \mathbb{C}\left\langle\left\langle X_{a} ; a \in \Sigma\right\rangle\right\rangle .
$$

This differential equation is also Fuchsian with singular points $\Sigma \cup\{\infty\}$, so for any $a, b \in \Sigma$ there is a unique local solution $H_{\Sigma}^{a b}(z)$ with the asymptotic property

$$
H_{\Sigma}^{a b}(z) \times\left(\frac{z-b}{a-b}\right)^{-X_{b}} \longrightarrow 1 \quad(z \rightarrow b) .
$$

Then we define the connection matrix $\Phi_{\Sigma}^{a b}\left(X_{c} ; c \in \Sigma\right) \in \mathbb{C}\left\langle\left\langle X_{c} ; c \in \Sigma\right\rangle\right\rangle$ by

$$
\Phi_{\Sigma}^{a b}\left(X_{c} ; c \in \Sigma\right)=H_{\Sigma}^{b a}(z)^{-1} H_{\Sigma}^{a b}(z) .
$$


This connection matrix is the counterpart of the KZ associator.

This article is organized as follows: In Section 2 the shuffle algebra w.r.t. (3) is defined and some properties are described. In Section 3 by using the shuffle algebra, we define the multiple polylogarithms and construct the solutions of (3). Comparing the two evaluations of the ratio of solutions, we obtain the linear relations of special values of multiple polylogarithms. In Section 4 we derive the dual and closed formula of the linear relations of special values of multiple polylogarithms from the symmetry of singular points of (3).

\section{Shuffle Algebra}

Following [8, 1], we introduce the shuffle algebra and its properties. We define a non-commutative polynomial algebra $\mathcal{A}_{\Sigma}:=\mathbb{C}\left\langle x_{a} ; a \in \Sigma\right\rangle$, and call the elements $\left\{x_{a} ; a \in \Sigma\right\}$ letters and the monomials in $\mathcal{A}_{\Sigma}$ words. The weight of the word is defined as the total number of letters which appear in it. In this algebra we define the shuffle product "ш" recursively as follows:

1. $w ш 1=1 ш w=w$,

2. $l_{1} w_{1}$ ш $l_{2} w_{2}=l_{1}\left(w_{1} ш l_{2} w_{2}\right)+l_{2}\left(l_{1} w_{1} ш w_{2}\right)$,

where $l_{1}$ and $l_{2}$ are letters, and $w_{1}, w_{2}$ and $w$ are words. We regard $\mathcal{A}_{\Sigma}=$ $\left(\mathcal{A}_{\Sigma},+, \boldsymbol{w}\right)$ and call it the "shuffle algebra". For example the $n$-th power of a letter $x_{a}$ is computed as

$$
x_{a}^{ш n}=\underbrace{x_{a} ш \cdots ш x_{a}}_{n}=n ! x_{a}^{n} .
$$

Fix any two elements $a$ and $b \in \Sigma$ and let " $\prec$ " be a total order of $\Sigma$ with the maximum element $a$ and the minimum element $b$. From this order, the words in $\mathcal{A}_{\Sigma}$ are totally ordered by the lexicographic order. The Lyndon words $\mathfrak{L}=\mathfrak{L}_{\Sigma, \prec} \subset \mathcal{A}_{\Sigma}$ are defined as follows:

$\mathfrak{L}=\left\{w \neq 1 \in \mathcal{A}_{\Sigma}\right.$ : word $\mid$ for any non-trivial decomposition $\left.w=u v, w \prec v\right\}$.

In particular $\mathfrak{L} \supset\left\{x_{a}\right\}_{a \in \Sigma}$ and Lyndon words which start from $x_{a}$ (respectively ends at $x_{b}$ ) are only $x_{a}$ (respectively $x_{b}$ ). Then the shuffle algebra $\mathcal{A}_{\Sigma}$ is the commutative polynomial algebra $\mathbb{C}[\mathfrak{L}]([10]$ Theorem 6.1 .). We define 
subalgebras of $\mathcal{A}_{\Sigma}$ as

$$
\begin{aligned}
& \mathcal{A}_{\Sigma}^{b}:=\mathbb{C}\left[\mathfrak{L} \backslash\left\{x_{b}\right\}\right]=\mathbb{C} .1 \oplus \bigoplus_{\substack{l \in \Sigma \\
l \neq b}} \mathcal{A}_{\Sigma} x_{l}, \\
& \mathcal{A}_{\Sigma}^{a b}:=\mathbb{C}\left[\mathfrak{L} \backslash\left\{x_{a}, x_{b}\right\}\right]=\mathbb{C} .1 \oplus \bigoplus_{\substack{l_{1}, l_{2} \in \Sigma \\
l_{1} \neq a, l_{2} \neq b}} x_{l_{1}} \mathcal{A}_{\Sigma} x_{l_{2}} .
\end{aligned}
$$

Because $x_{a}$ and $x_{b} \in L, \mathcal{A}_{\Sigma}$ can be written as follows:

$$
\begin{aligned}
\mathcal{A}_{\Sigma} & =\mathcal{A}_{\Sigma}^{b}\left[x_{b}\right]:=\bigoplus_{j=0}^{\infty} \mathcal{A}_{\Sigma}^{b} ш x_{b}^{ш j} \\
& =\mathcal{A}_{\Sigma}^{a b}\left[x_{a}, x_{b}\right]:=\bigoplus_{i, j=0}^{\infty} x_{a}^{ш i} ш \mathcal{A}_{\Sigma}^{a b} ш x_{b}^{ш j} .
\end{aligned}
$$

Using these decompositions, we define $\mathrm{reg}^{b}: \mathcal{A}_{\Sigma} \rightarrow \mathcal{A}_{\Sigma}^{b}$ and reg ${ }^{a b}: \mathcal{A}_{\Sigma} \rightarrow \mathcal{A}_{\Sigma}^{a b}$ to be the maps sending any word to its constant term of $\mathcal{A}_{\Sigma}^{b}\left[x_{b}\right]$ and $\mathcal{A}_{\Sigma}^{a b}\left[x_{a}, x_{b}\right]$ respectively. By definition, $\operatorname{reg}^{b}$ and $\operatorname{reg}^{a b}$ are $\boldsymbol{w}$-homomorphisms.

Proposition 1 ([8]). For $w=w_{b} x_{b}^{n}=x_{a}^{m} w_{a b} x_{b}^{n} \in \mathcal{A}_{\Sigma}\left(w_{b} \in \mathcal{A}_{\Sigma}^{b}, w_{a b} \in \mathcal{A}_{\Sigma}^{a b}\right)$ we have

$$
\begin{aligned}
& \operatorname{reg}^{b}\left(w_{b} x_{b}^{n}\right)=\sum_{j=0}^{n}(-1)^{j} w_{b} x_{b}^{n-j} \text { ш } x_{b}^{j}, \\
& \operatorname{reg}^{a b}\left(x_{a}^{m} w_{a b} x_{b}^{n}\right)=\sum_{i=0}^{m} \sum_{j=0}^{n}(-1)^{i+j} x_{a}^{i} ш x_{a}^{m-i} w_{b} x_{b}^{n-j} ш x_{b}^{j}, \\
& w=\sum_{j=0}^{n} \operatorname{reg}^{b}\left(w_{b} x_{b}^{n-j}\right) ш x_{b}^{j}=\sum_{i=0}^{m} \sum_{j=0}^{n} x_{a}^{i} \operatorname{reg}^{a b}\left(x_{a}^{m-i} w_{a b} x_{b}^{n-j}\right) ш x_{b}^{j} .
\end{aligned}
$$

Prepare the generating function of all words of $\mathcal{A}_{\Sigma}$ in the non-commutative power series algebra $\mathcal{A}_{\Sigma}\left\langle\left\langle X_{a} ; a \in \Sigma\right\rangle\right\rangle$

$$
\begin{aligned}
\sum_{W} w W=1 & +x_{a_{0}} X_{a_{0}}+x_{a_{1}} X_{a_{1}}+x_{a_{2}} X_{a_{2}} \cdots \\
& +x_{a_{0}} x_{a_{0}} X_{a_{0}} X_{a_{0}}+x_{a_{0}} x_{a_{1}} X_{a_{0}} X_{a_{1}}+x_{a_{0}} x_{a_{2}} X_{a_{0}} X_{a_{2}}+\cdots \\
& +x_{a_{1}} x_{a_{0}} X_{a_{1}} X_{a_{0}}+x_{a_{1}} x_{a_{1}} X_{a_{1}} X_{a_{1}}+x_{a_{1}} x_{a_{2}} X_{a_{1}} X_{a_{2}}+\cdots
\end{aligned}
$$

where the sum is taken over all words in $\mathcal{A}_{\Sigma}\left\langle\left\langle X_{a} ; a \in \Sigma\right\rangle\right\rangle$ and $W$ is the capitalization of $w$. Noting (4) and using this generating function, (9) can 
be expressed by

$$
\begin{aligned}
\sum_{W} w W & =\left(\sum_{W} \operatorname{reg}^{b}(w) W\right) \times \exp \left(x_{b} X_{b}\right) \\
& =\exp \left(x_{a} X_{a}\right) \times\left(\sum_{W} \operatorname{reg}^{a b}(w) W\right) \times \exp \left(x_{b} X_{b}\right),
\end{aligned}
$$

where $\exp (x X)$ means

$$
\exp (x X)=\sum_{n=0}^{\infty} \frac{x^{ш n}}{n !} X^{n}=\sum_{n=0}^{\infty} x^{n} X^{n} .
$$

The inverse $\left(\sum_{W} w W\right)^{-1}$ is

$$
\left(\sum_{W} w W\right)^{-1}=\sum_{W} S(w) W=\sum_{W} w S(W)
$$

where $S$ is an anti-involution w.r.t. the ordinary product defined by $S: x_{a} \mapsto$ $-x_{a}, X_{a} \mapsto-X_{a}$. In general any homomorphism or anti-homomorphism w.r.t. ordinary product is a homomorphism w.r.t. $\boldsymbol{~ ш . ~ S o ~} S$ is also a $ш-$ homomorphism.

\section{Multiple Polylogarithms}

For a word $w=x_{b}^{k_{1}-1} x_{c_{1}} \cdots x_{b}^{k_{r-1}-1} x_{c_{r-1}} x_{b}^{k_{r}-1} x_{c_{r}} \in \mathcal{A}_{\Sigma}^{b}\left(b \neq c_{i} \in \Sigma\right)$ we define the multiple polylogarithm (of one variable) $\mathrm{Li}_{\Sigma}^{b}(w ; z)$ by

$$
\begin{aligned}
\operatorname{Li}_{\Sigma}^{b}(1 ; z) & :=1, \\
\operatorname{Li}_{\Sigma}^{b}(w ; z) & :=(-1)^{r} \lambda\left(\begin{array}{cccc}
k_{1} & \cdots & k_{r-1} & k_{r} \\
\frac{c_{1}-b}{z-b} & \ldots & \frac{c_{r-1}-b}{z-b} & \frac{c_{r}-b}{z-b}
\end{array}\right) \\
& =(-1)^{r} \sum_{m_{1}>\cdots>m_{r}>0} \frac{\left(\frac{z-b}{c_{1}-b}\right)^{m_{1}-m_{2}} \cdots\left(\frac{z-b}{c_{r-1}-b}\right)^{m_{r-1}-m_{r}}\left(\frac{z-b}{c_{r}-b}\right)^{m_{r}}}{m_{1}^{k_{1}} \cdots m_{r-1}^{k_{r-1}} m_{r}^{k_{r}}}
\end{aligned}
$$

where the series converges absolutely for $|z-b|<\min _{c \in \Sigma}|c-b|$. If the weight of $w$ is greater than or equal to $1, \operatorname{Li}_{\Sigma}^{b}(w ; b)=0$. Moreover $\mathrm{Li}$ is extended linearly w.r.t. $w$. For example

$$
\operatorname{Li}_{\Sigma}^{b}\left(x_{c} ; z\right)=(-1) \sum_{m=1}^{\infty} \frac{\left(\frac{z-b}{c-b}\right)^{m}}{m}=\log \left(1-\frac{z-b}{c-b}\right)=\log \left(\frac{z-c}{b-c}\right) .
$$


$\operatorname{Li}_{\Sigma}^{b}(w ; z)$ is easily expressed as the iterated integral

$$
\begin{aligned}
& \operatorname{Li}_{\Sigma}^{b}(w ; z)=\underbrace{\int_{b}^{z} \frac{d z}{z-b} \cdots \int_{b}^{z} \frac{d z}{z-b} \int_{b}^{z} \frac{d z}{z-c_{1}}}_{k_{1}} \cdots \\
& \cdots \underbrace{\int_{b}^{z} \frac{d z}{z-b} \cdots \int_{b}^{z} \frac{d z}{z-b} \int_{b}^{z} \frac{d z}{z-c_{r-1}}}_{k_{r}-1} \underbrace{\int_{b}^{z} \frac{d z}{z-b} \cdots \int_{b}^{z} \frac{d z}{z-b} \int_{b}^{z} \frac{d z}{z-c_{r}}}_{k_{r}} .
\end{aligned}
$$

From this expression $\operatorname{Li}_{\Sigma}^{b}(w ; z)$ can be analytically continued to $\mathbb{C} \backslash \Sigma$ and the derivative is

$$
\frac{d}{d z} \operatorname{Li}_{\Sigma}^{b}\left(x_{c} w ; z\right)=\frac{1}{z-c} \operatorname{Li}_{\Sigma}^{b}(w ; z)
$$

for any letter $c$ and any word $w \in \mathcal{A}_{\Sigma}^{b}$. By using an induction w.r.t. the sum of weight of words, we obtain the next proposition.

Proposition 2. For any words $w_{1}, w_{2} \in \mathcal{A}_{\Sigma}^{b}$,

$$
\operatorname{Li}_{\Sigma}^{b}\left(w_{1} ; z\right) \operatorname{Li}_{\Sigma}^{b}\left(w_{2} ; z\right)=\operatorname{Li}_{\Sigma}^{b}\left(w_{1} ш w_{2} ; z\right) .
$$

This proposition inspires us to define $\operatorname{Li}_{\Sigma}^{a b}(w ; z)$ for any word $w \in \mathcal{A}_{\Sigma}$ by

$$
\begin{gathered}
\operatorname{Li}_{\Sigma}^{a b}(w ; z):=\operatorname{Li}_{\Sigma}^{b}(w ; z) \quad \text { for } w \in \mathcal{A}_{\Sigma}^{b}, \\
\operatorname{Li}_{\Sigma}^{a b}\left(x_{b} ; z\right):=\log \left(\frac{z-b}{a-b}\right)=\operatorname{Li}_{\Sigma}^{a}\left(x_{b} ; z\right),
\end{gathered}
$$

and extend as a $\boldsymbol{w}$-homomorphism w.r.t. $\mathcal{A}_{\Sigma}$. It is well-defined because of (5).

\section{Theorem 3.}

$$
H_{\Sigma}^{a b}(z)=\sum_{W} \operatorname{Li}_{\Sigma}^{a b}(w ; z) W
$$

Proof. Applying $\operatorname{Li}_{\Sigma}^{a b}(\cdot ; z)$ to (10) we have

$$
\begin{aligned}
\sum_{W} \operatorname{Li}_{\Sigma}^{a b}(w ; z) W & =\left(\sum_{W} \operatorname{Li}_{\Sigma}^{b}\left(\operatorname{reg}^{b}(w) ; z\right) W\right) \times \exp \left(\operatorname{Li}_{\Sigma}^{a b}\left(x_{b} ; z\right) X_{b}\right) \\
& =\left(\sum_{W} \operatorname{Li}_{\Sigma}^{b}\left(\operatorname{reg}^{b}(w) ; z\right) W\right) \times\left(\frac{z-b}{a-b}\right)^{X_{b}} .
\end{aligned}
$$


So the asymptotic property is satisfied. We must show that this series is the solution of (31), i.e. (13) holds for any $w \in \mathcal{A}_{\Sigma}$. Applying (77) to the word $x_{c} w_{b} x_{b}^{n}\left(w_{b} \in \mathcal{A}_{\Sigma}^{b}\right)$ we have

$$
\begin{aligned}
\operatorname{reg}^{b}\left(x_{c} w_{b} x_{b}^{n}\right) & =\sum_{j=0}^{n}(-1)^{j} x_{c} w_{b} x_{b}^{n-j} \text { ш } x_{b}^{j} \\
& =x_{c} \sum_{j=0}^{n}(-1)^{j} w_{b} x_{b}^{n-j} ш x_{b}^{j}+x_{b} \sum_{j=1}^{n}(-1)^{j} x_{c} w_{b} x_{b}^{n-j} \boldsymbol{ш} x_{b}^{j-1} \\
& =x_{c} \operatorname{reg}^{b}\left(w_{b} x_{b}^{n}\right)-x_{b} \operatorname{reg}^{b}\left(x_{c} w_{b} x_{b}^{n-1}\right) .
\end{aligned}
$$

With this formula and (99), we can calculate the derivative of $\operatorname{Li}_{\Sigma}^{a b}\left(x_{c} w_{b} x_{b}^{n} ; z\right)$ as follows:

$$
\begin{aligned}
& \frac{d}{d z} \operatorname{Li}_{\Sigma}^{a b}\left(x_{c} w_{b} x_{b}^{n} ; z\right) \\
& =\frac{d}{d z} \sum_{j=0}^{n} \operatorname{Li}_{\Sigma}^{a b}\left(\operatorname{reg}^{b}\left(x_{c} w_{b} x_{b}^{n-j}\right) ; z\right) \operatorname{Li}_{\Sigma}^{a b}\left(x_{b}^{j} ; z\right) \\
& =\frac{1}{z-c} \operatorname{Li}_{\Sigma}^{a b}\left(\operatorname{reg}^{b}\left(w_{b} x_{b}^{n}\right) ; z\right)-\frac{1}{z-b} \operatorname{Li}_{\Sigma}^{a b}\left(\operatorname{reg}^{b}\left(w_{b} x_{b}^{n-1}\right) ; z\right) \\
& \quad+\sum_{j=1}^{n-1}\left\{\frac{1}{z-c} \operatorname{Li}_{\Sigma}^{a b}\left(\operatorname{reg}^{b}\left(w_{b} x_{b}^{n-j}\right) ; z\right) \operatorname{Li}_{\Sigma}^{a b}\left(x_{b}^{j} ; z\right)\right. \\
& \quad-\frac{1}{z-b} \operatorname{Li}_{\Sigma}^{a b}\left(\operatorname{reg}^{b}\left(x_{c} w_{b} x_{b}^{n-j-1}\right) ; z\right) \operatorname{Li}_{\Sigma}^{a b}\left(x_{b}^{j} ; z\right) \\
& \left.\quad+\frac{1}{z-b} \operatorname{Li}_{\Sigma}^{a b}\left(\operatorname{reg}^{b}\left(x_{c} w_{b} x_{b}^{n-j}\right) ; z\right) \operatorname{Li}_{\Sigma}^{a b}\left(x_{b}^{j-1} ; z\right)\right\} \\
& \quad+\frac{1}{z-c} \operatorname{Li}_{\Sigma}^{a b}\left(\operatorname{reg}^{b}\left(w_{b}\right) ; z\right) \operatorname{Li}_{\Sigma}^{a b}\left(x_{b}^{n} ; z\right) \\
& \quad \frac{1}{z-b} \operatorname{Li}_{\Sigma}^{a b}\left(\operatorname{reg}^{b}\left(x_{c} w_{b}\right) ; z\right) \operatorname{Li}_{\Sigma}^{a b}\left(x_{b}^{n-1} ; z\right) \\
& =\frac{1}{z-c} \sum_{j=0}^{n} \operatorname{Li}_{\Sigma}^{a b}\left(w_{b}^{a b}\left(\operatorname{reg}_{b}^{n}\left(w_{b} x_{b}^{n-j}\right) ; z\right) \operatorname{Li}_{\Sigma}^{a b}\left(x_{b}^{j} ; z\right)\right.
\end{aligned}
$$

Using (11), (12) and (15) $H_{\Sigma}^{a b}(z)$ also can be written as

$$
H_{\Sigma}^{a b}(z)=\left(\frac{z-a}{b-a}\right)^{X_{a}}\left(\sum_{W} \operatorname{Li}_{\Sigma}^{a b}\left(\operatorname{reg}^{a b}(w) ; z\right) W\right)\left(\frac{z-b}{a-b}\right)^{X_{b}},
$$


and in the same way $H_{\Sigma}^{b a}(z)$ is

$$
H_{\Sigma}^{b a}(z)=\left(\frac{z-b}{a-b}\right)^{X_{b}}\left(\sum_{W} \operatorname{Li}_{\Sigma}^{b a}\left(\operatorname{reg}^{b a}(w) ; z\right) W\right)\left(\frac{z-a}{b-a}\right)^{X_{a}} .
$$

Let $a$ be one of the nearest points of $\Sigma$ to $b$. Then the ratio of these two solutions is

$$
\begin{aligned}
H_{\Sigma}^{b a}(z)^{-1} H_{\Sigma}^{a b}(z) & \left(\sum_{W} \operatorname{Li}_{\Sigma}^{b a}(S(w) ; z) W\right)\left(\sum_{W} \operatorname{Li}_{\Sigma}^{a b}(w ; z) W\right) \\
= & \sum_{W}\left(\sum_{w_{1} w_{2}=w} \operatorname{Li}_{\Sigma}^{b a}\left(S\left(w_{1}\right) ; z\right) \operatorname{Li}_{\Sigma}^{a b}\left(w_{2} ; z\right)\right) W \\
= & \left(\frac{z-a}{b-a}\right)^{-X_{a}}\left(\sum_{W} \operatorname{Li}_{\Sigma}^{b a}\left(\operatorname{reg}^{b a} \circ S(w) ; z\right) W\right)\left(\frac{z-b}{a-b}\right)^{-X_{b}} \\
& \quad \times\left(\frac{z-a}{b-a}\right)^{X_{a}}\left(\sum_{W} \operatorname{Li}_{\Sigma}^{a b}\left(\operatorname{reg}^{a b}(w) ; z\right) W\right)\left(\frac{z-b}{a-b}\right)^{X_{b}} .
\end{aligned}
$$

On the other hand, for any $w=x_{b}^{k_{1}-1} x_{c_{1}} \cdots x_{b}^{k_{r}-1} x_{c_{r}} \in \mathcal{A}^{a b}, \operatorname{Li}_{\Sigma}^{a b}(w ; z)$ can be evaluated at $z=a$. We define $\mathcal{L}_{\Sigma}^{a b}: \mathcal{A}_{\Sigma}^{a b} \rightarrow \mathbb{C}$ to be this evaluation:

$$
\begin{aligned}
& \mathcal{L}_{\Sigma}^{a b}(w):=\lim _{t \rightarrow 1} \operatorname{Li}_{\Sigma}^{a b}(w ; t a+(1-t) b) \\
& =(-1)^{r} \sum_{m_{1}=r}^{\infty} \sum_{m_{1}>m_{2}>\cdots>m_{r}>0} \frac{\left(\frac{a-b}{c_{1}-b}\right)^{m_{1}-m_{2}} \cdots\left(\frac{a-b}{c_{r-1}-b}\right)^{m_{r-1}-m_{r}}\left(\frac{a-b}{c_{r}-b}\right)^{m_{r}}}{m_{1}^{k_{1}} \cdots m_{r-1}^{k_{r}-1} m_{r}^{k_{r}}} \\
& =(-1)^{r} \lambda\left(\begin{array}{ccc}
k_{1} & \cdots & k_{r} \\
\frac{c_{1}-b}{a-b} & \cdots & \frac{c_{r}-b}{a-b}
\end{array}\right) .
\end{aligned}
$$

$\mathcal{L}_{\Sigma}^{a b}$ is obviously a w-homomorphism. Tending $z$ to $a$ in (21) we have

$$
\Phi_{\Sigma}^{a b}\left(X_{c} ; c \in \Sigma\right)=\sum_{W} \mathcal{L}_{\Sigma}^{a b}\left(\operatorname{reg}^{a b}(w)\right) W
$$

The coefficients of (20) yield the functional equation

$$
\sum_{w_{1} w_{2}=w} \operatorname{Li}_{\Sigma}^{b a}\left(S\left(w_{1}\right) ; z\right) \operatorname{Li}_{\Sigma}^{a b}\left(w_{2} ; z\right)=\mathcal{L}_{\Sigma}^{a b}\left(\operatorname{reg}^{a b}(w)\right)
$$


for any word $w \in \mathcal{A}_{\Sigma}$. We call this functional equation "Euler's inversion formula". If $a$ and $b$ are the nearest points to each other, we can also evaluate the ratio (21) at $b$ and we have

$$
\Phi_{\Sigma}^{a b}\left(X_{c} ; c \in \Sigma\right)=\sum_{W} \mathcal{L}_{\Sigma}^{b a}\left(\operatorname{reg}^{b a} \circ S(w)\right) W .
$$

Consequently for any word $w \in \mathcal{A}_{\Sigma}$ we obtain

$$
\mathcal{L}_{\Sigma}^{a b}\left(\operatorname{reg}^{a b}(w)\right)=\mathcal{L}_{\Sigma}^{a b}\left(\operatorname{reg}^{b a}(S(w))\right) .
$$

We call this equation the "duality formula".

Theorem 4. For any $b \in \Sigma$ and one of the nearest points $a \in \Sigma$ to $b$,

$$
\Phi_{\Sigma}^{a b}\left(X_{c} ; c \in \Sigma\right)=\sum_{W} \mathcal{L}_{\Sigma}^{a b}\left(\operatorname{reg}^{a b}(w)\right) W .
$$

In particular for any word $w \in \mathcal{A}_{\Sigma}^{a b}$ we have

$$
\sum_{w_{1} w_{2}=w} \operatorname{Li}_{\Sigma}^{b a}\left(S\left(w_{1}\right) ; z\right) \operatorname{Li}_{\Sigma}^{a b}\left(w_{2} ; z\right)=\mathcal{L}_{\Sigma}^{a b}(w) .
$$

Moreover, if $b$ is one of the nearest points to $a$, we have

$$
\mathcal{L}_{\Sigma}^{b a}(S(w))=\mathcal{L}_{\Sigma}^{a b}(w) .
$$

Example 1. Set the singular points to be $\Sigma=\{0,1\}, a=1$ and $b=0$. Then for positive integers $a_{i}, b_{i}(i=1, \ldots, s)$ the coefficients of the connection matrix are written as follows:

$$
\begin{aligned}
& \mathcal{L}_{\{0,1\}}^{10}\left(x_{0}^{a_{1}} x_{1}^{b_{1}} x_{0}^{a_{2}} x_{1}^{b_{2}} \cdots x_{0}^{a_{s}} x_{1}^{b_{s}}\right) \\
& =(-)^{\sum_{i=1}^{s} b_{i}} \zeta(\underbrace{a_{1}+1,1, \ldots, 1}_{b_{1}}, \underbrace{a_{2}+1,1, \ldots, 1}_{b_{2}}, \ldots, \underbrace{a_{s}+1,1, \ldots, 1}_{b_{s}}), \\
& \mathcal{L}_{\{0,1\}}^{01}\left(S\left(x_{0}^{a_{1}} x_{1}^{b_{1}} x_{0}^{a_{2}} x_{1}^{b_{2}} \cdots x_{0}^{a_{s}} x_{1}^{b_{s}}\right)\right) \\
& =(-)^{\sum_{i=1}^{s} a_{i}+b_{i}} \mathcal{L}_{\{0,1\}}^{01}\left(x_{1}^{b_{s}} x_{0}^{a_{s}} \cdots x_{1}^{b_{2}} x_{0}^{a_{2}} x_{1}^{b_{1}} x_{0}^{a_{1}}\right) \\
& =(-)^{\sum_{i=1}^{s} b_{i}} \zeta(\underbrace{b_{s}+1,1, \ldots, 1}_{a_{s}}, \ldots, \underbrace{b_{2}+1,1, \ldots, 1}_{a_{2}}, \underbrace{b_{1}+1,1, \ldots, 1}_{a_{1}}) .
\end{aligned}
$$

From (22) we have the equation

$$
\begin{aligned}
\zeta(\underbrace{a_{1}+1,1, \ldots, 1}_{b_{1}}, \underbrace{a_{2}+1,1, \ldots, 1}_{b_{2}}, \ldots, \underbrace{a_{s}+1,1, \ldots, 1}_{b_{s}}) \\
=\zeta(\underbrace{b_{s}+1,1, \ldots, 1}_{a_{s}}, \ldots, \underbrace{b_{2}+1,1, \ldots, 1}_{a_{2}}, \underbrace{b_{1}+1,1, \ldots, 1}_{a_{1}}),
\end{aligned}
$$


this is the duality formula for multiple zeta values ([1]).

Euler's inversion formula for $x_{0} x_{1}$ is

$$
\begin{aligned}
\operatorname{Li}_{\{0,1\}}^{01}\left(x_{1} x_{0} ; z\right)+\operatorname{Li}_{\{0,1\}}^{01}\left(x_{0} ; z\right) \operatorname{Li}_{\{0,1\}}^{10}\left(x_{1} ; z\right) & \\
& +\operatorname{Li}_{\{0,1\}}^{10}\left(x_{0} x_{1} ; z\right)=\mathcal{L}_{\{0,1\}}^{10}\left(x_{0} x_{1}\right),
\end{aligned}
$$

or equivalently

$$
\operatorname{Li}_{2}(1-z)+\log (z) \log (1-z)+\operatorname{Li}_{2}(z)=\zeta(2) .
$$

Here $\operatorname{Li}_{2}(z)=\sum_{n=1}^{\infty} z^{n} / n^{2}$ is Euler's dilogarithm. This formula is well-known as Euler's inversion formula for the dilogarithm.

Example 2. Set $\Sigma=\{0,1,-1\}, a=1$ and $b=0$. Any word $w_{10} \in \mathcal{A}_{\{0,1,-1\}}^{10}$ can be written as

$$
w_{10}=x_{0}^{k_{1}-1} x_{d_{1}} x_{0}^{k_{2}-1} x_{d_{2}} \cdots x_{0}^{k_{r-1}-1} x_{d_{r-1}} x_{0}^{k_{r}-1} x_{d_{r}} \quad\left(d_{i}= \pm 1\right),
$$

and $S\left(w_{01}\right)$ also can be written as

$$
S\left(w_{10}\right)=(-)^{\sum_{i=1}^{r^{\prime}} k_{i}^{\prime}} x_{1}^{k_{1}^{\prime}-1} x_{e_{1}} x_{2}^{k_{2}^{\prime}-1} x_{e_{2}} \cdots x_{1}^{k_{r^{\prime}-1}^{\prime}-1} x_{e_{r^{\prime}-1}} x_{1}^{k_{r^{\prime}}^{\prime}-1} x_{e_{r^{\prime}}}\left(e_{j}=0,-1\right) .
$$

Then the duality formula gives us the equation

$$
\begin{gathered}
(-)^{\left|w_{01}\right|_{x-1}} \sum_{m_{1}>\cdots>m_{r-1}>m_{r}} \frac{(-1)^{d_{1}\left(m_{1}-m_{2}\right)+d_{2}\left(m_{2}-m_{3}\right)+\cdots+d_{r-1}\left(m_{r-1}-m_{r}\right)+d_{r} m_{r}}}{m_{1}^{k_{1}} m_{2}^{k_{2}} \cdots m_{r-1}^{k_{r-1}} m_{r}^{k_{r}}} \\
=\sum_{m_{1}>\cdots>m_{r^{\prime}-1}>m_{r^{\prime}}} \frac{\left(\frac{1}{2}\right)^{-e_{1}\left(m_{1}-m_{2}\right)-e_{2}\left(m_{2}-m_{3}\right)-\cdots-e_{r^{\prime}-1}\left(m_{r^{\prime}-1}-m_{r^{\prime}}\right)-e_{r^{\prime}} m_{r^{\prime}}}}{m_{1}^{k_{1}^{\prime}} m_{2}^{k_{2}^{\prime}} \cdots m_{r^{\prime}-1}^{k_{r^{\prime}-1}} m_{r^{\prime}}^{k_{r^{\prime}}^{\prime}}},
\end{gathered}
$$

where $\left|w_{01}\right|_{x_{-1}}$ is the total number of factors of $x_{-1}$ in $w_{01}$. For example, the duality formula w.r.t. $x_{-1}$ is

$$
-(-\log (2))=-\sum_{m=1}^{\infty} \frac{(-)^{m}}{m}=\sum_{m=1}^{\infty} \frac{1}{m}\left(\frac{1}{2}\right)^{m}=-\log \left(1-\frac{1}{2}\right),
$$

and the duality formula w.r.t. $x_{0} x_{1} x_{-1}$ is

$$
-\sum_{m_{1}>m_{2}} \frac{(-)^{m_{2}}}{m_{1}^{2} m_{2}}=\sum_{m_{1}>m_{2}} \frac{1}{m_{1} m_{2}^{2}}\left(\frac{1}{2}\right)^{m_{1}-m_{2}} .
$$

Equations (24) and (25) are respectively the duality formulas of Examples 6.8 and 6.1 of [2]. The left hand side of (23) is the multiple $L$-value of modulus 2 but unfortunately the right hand side is not. 


\section{Duality from the symmetry of $\Sigma$}

For singular points $\Sigma$, we consider the subgroup $G_{\Sigma}$ of the linear transforms $\operatorname{PSL}(2 ; \mathbb{C})$ preserving $\Sigma \cup\{\infty\}$. Defining the action of $\sigma \in G_{\Sigma}$ on the $x_{c} \in \mathcal{A}_{\Sigma}$ as

$$
\sigma\left(x_{c}\right)=x_{\sigma(c)}-x_{\sigma(\infty)}
$$

and extending as the homomorphism w.r.t. ordinary product (so $\sigma$ is a $\boldsymbol{~ ш - ~}$ homomorphism), $G_{\Sigma}$ acts on $\mathcal{A}_{\Sigma}$. (Here $x_{\infty}$ means 0 .) Then $G_{\Sigma}$ acts on $\operatorname{Li}_{\Sigma}^{a b}(w ; z)$ on the left and right by

$$
\begin{aligned}
& \left(\sigma \operatorname{Li}_{\Sigma}^{a b}\right)(w ; z)=\operatorname{Li}_{\Sigma}^{a b}\left(\sigma^{-1}(w) ; z\right), \\
& \left(\operatorname{Li}_{\Sigma}^{a b} \sigma\right)(w ; z)=\operatorname{Li}_{\Sigma}^{a b}(w ; \sigma(z)) .
\end{aligned}
$$

Proposition 5. For $\sigma, \tau \in G_{\Sigma}$, and $c \in \Sigma$ we have

$$
\begin{aligned}
\frac{d}{d z}\left(\tau \operatorname{Li}_{\Sigma}^{a b} \sigma\right) & \left(x_{c} w ; z\right) \\
& =\left\{\frac{1}{z-(\tau \circ \sigma)^{-1}(c)}-\frac{1}{z-(\tau \circ \sigma)^{-1}(\infty)}\right\}\left(\tau \operatorname{Li}_{\Sigma}^{a b} \sigma\right)(w ; z) .
\end{aligned}
$$

Proof. If $\tau(\infty) \notin\{c, \infty\}$

$$
\begin{aligned}
& \frac{d}{d z}\left(\tau \operatorname{Li}_{\Sigma}^{a b} \sigma\right)\left(x_{c} w ; z\right)=\frac{d}{d z} \operatorname{Li}_{\Sigma}^{a b}\left(\left(x_{\tau^{-1}(c)}-x_{\tau^{-1}(\infty)}\right) \tau^{-1}(w) ; \sigma(z)\right) \\
&=\left\{\frac{1}{z-\sigma^{-1}\left(\tau^{-1}(c)\right)}-\frac{1}{z-\sigma^{-1}(\infty)}\right\}\left(\tau \operatorname{Li}_{\Sigma}^{a b} \sigma\right)\left(x_{c} w ; z\right) \\
&-\left\{\frac{1}{z-\sigma^{-1}\left(\tau^{-1}(\infty)\right)}-\frac{1}{z-\sigma^{-1}(\infty)}\right\}\left(\tau \operatorname{Li}_{\Sigma}^{a b} \sigma\right)\left(x_{c} w ; z\right) \\
&=\left\{\frac{1}{z-(\tau \circ \sigma)^{-1}(c)}-\frac{1}{z-(\tau \circ \sigma)^{-1}(\infty)}\right\}\left(\tau \operatorname{Li}_{\Sigma}^{a b} \sigma\right)(w ; z) .
\end{aligned}
$$

If $\tau(\infty)=c$,

$$
\begin{aligned}
\frac{d}{d z} & \left(\tau \operatorname{Li}_{\Sigma}^{a b} \sigma\right)\left(x_{c} w ; z\right)=-\frac{d}{d z} \operatorname{Li}_{\Sigma}^{a b}\left(x_{\tau^{-1}(\infty)} \tau^{-1}(w) ; \sigma(z)\right) \\
& =-\left\{\frac{1}{z-\sigma^{-1}\left(\tau^{-1}(\infty)\right)}-\frac{1}{z-\sigma^{-1}(\infty)}\right\}\left(\tau \operatorname{Li}_{\Sigma}^{a b} \sigma\right)\left(x_{c} w ; z\right) \\
& =\left\{\frac{1}{z-(\tau \circ \sigma)^{-1}(c)}-\frac{1}{z-(\tau \circ \sigma)^{-1}(\infty)}\right\}\left(\tau \operatorname{Li}_{\Sigma}^{a b} \sigma\right)(w ; z) .
\end{aligned}
$$

In the same way the case $\tau(\infty)=\infty$ can be proved. 
In particular take $\tau=\sigma^{-1}$ and we have

$$
\frac{d}{d z}\left(\sigma^{-1} \operatorname{Li}_{\Sigma}^{a b} \sigma\right)\left(x_{c} w ; z\right)=\frac{1}{z-c}\left(\sigma^{-1} \operatorname{Li}_{\Sigma}^{a b} \sigma\right)(w ; z)
$$

i.e.

$$
\left(\sigma^{-1} H_{\Sigma}^{a b} \sigma\right)(z)=\sum_{W}\left(\sigma^{-1} \operatorname{Li}_{\Sigma}^{a b} \sigma\right)(w ; z) W
$$

becomes the solution of (3) again. To determine this function, we need to know the asymptotic property around some point.

$G_{\Sigma}$ also acts on $\mathbb{C}\left\langle\left\langle X_{c} ; c \in \Sigma\right\rangle\right\rangle$ as

$$
\sigma\left(X_{c}\right)=X_{\sigma(c)}
$$

where $X_{\infty}=-\sum_{c \in \Sigma} X_{c}$. Then

$$
\begin{aligned}
\sum_{W} \sigma(w) W & =\sum_{W} w \sigma^{-1}(W) \\
& =\exp \left(x_{a} \sigma^{-1}\left(X_{a}\right)\right)\left(\sum_{W} \operatorname{reg}^{a b}(w) \sigma^{-1}(W)\right) \exp \left(x_{b} \sigma^{-1}\left(X_{b}\right)\right) .
\end{aligned}
$$

Applying $\operatorname{Li}_{\Sigma}^{a b}(\cdot ; z)$ to this equation, we get

$$
\begin{aligned}
& \left(\sigma^{-1} H_{\Sigma}^{a b} \sigma\right)(z)=\sum_{W} \operatorname{Li}_{\Sigma}^{a b}(w ; \sigma(z)) \sigma^{-1}(W) \\
& =\left(\frac{\sigma(z)-a}{b-a}\right)^{X_{\sigma^{-1}(a)}}\left(\sum_{W} \operatorname{Li}_{\Sigma}^{a b}\left(\operatorname{reg}^{a b}(w) ; \sigma(z)\right) \sigma^{-1}(W)\right)\left(\frac{\sigma(z)-b}{a-b}\right)^{X_{\sigma^{-1}(b)}} .
\end{aligned}
$$

In particular assume that $\sigma(a)=b$ and $\sigma(b)=a$. Then $\sigma$ is expressed as

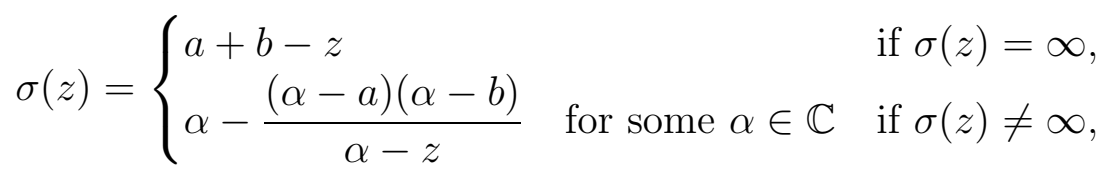

and it is easy to check that $\sigma$ is involutive. Here $\alpha$ is the image of $\infty$. Under this assumption, we have

$$
\begin{array}{r}
\left(\sigma^{-1} H_{\Sigma}^{a b} \sigma\right)(z) \\
=\left(\frac{z-b}{a-b} \frac{a-\sigma(\infty)}{z-\sigma(\infty)}\right)^{X_{b}}\left(\sum_{W} \operatorname{Li}_{\Sigma}^{a b}\left(\operatorname{reg}^{a b}(w) ; \sigma(z)\right) \sigma^{-1}(W)\right) \\
\times\left(\frac{z-a}{b-a} \frac{b-\sigma(\infty)}{z-\sigma(\infty)}\right)^{X_{a}},
\end{array}
$$


where

$$
\frac{a-\sigma(\infty)}{z-\sigma(\infty)}=\frac{b-\sigma(\infty)}{z-\sigma(\infty)}=1 \quad \text { if } \sigma(\infty)=\infty
$$

Thus we have

$$
\left(\sigma^{-1} H_{\Sigma}^{a b} \sigma\right)(z)\left(\frac{z-a}{b-a}\right)^{-X_{a}} \longrightarrow\left(\frac{b-\sigma(\infty)}{a-\sigma(\infty)}\right)^{X_{a}} \quad(z \rightarrow a),
$$

in other words

$$
\left(\sigma^{-1} H_{\Sigma}^{a b} \sigma\right)(z)\left(\frac{a-\sigma(\infty)}{b-\sigma(\infty)}\right)^{X_{a}}=H_{\Sigma}^{b a}(z)
$$

Moreover assume that $a$ and $b$ are the nearest points to each other in $\Sigma$. Then by the above expression of $H_{\Sigma}^{b a}(z)$, we can compute the connection matrix as

$$
\begin{aligned}
& \Phi_{\Sigma}^{a b}\left(X_{c} ; c \in \Sigma\right)=H_{\Sigma}^{b a}(z)^{-1} H_{\Sigma}^{a b}(z) \\
& =\left(\frac{b-\sigma(\infty)}{a-\sigma(\infty)}\right)^{X_{a}} \sum_{W}\left(\sum_{w_{1} w_{2}=w}\left(\sigma^{-1} \operatorname{Li}_{\Sigma}^{a b} \sigma\right)\left(S\left(w_{1}\right) ; z\right) \operatorname{Li}_{\Sigma}^{a b}\left(w_{2} ; z\right)\right) W \\
& =\left(\frac{b-a}{z-a} \frac{z-\sigma(\infty)}{a-\sigma(\infty)}\right)^{X_{a}}\left(\sum_{W} \operatorname{Li}_{\Sigma}^{a b}\left(\operatorname{reg}^{a b} \circ \sigma \circ S(w) ; \sigma(z)\right) W\right) \\
& \times\left(\frac{a-b}{z-b} \frac{z-\sigma(\infty)}{a-\sigma(\infty)}\right)^{X_{b}} \\
& \quad \times\left(\frac{z-a}{b-a}\right)^{X_{a}}\left(\sum_{W} \operatorname{Li}_{\Sigma}^{a b}\left(\operatorname{reg}^{a b}(w) ; z\right) W\right)\left(\frac{z-b}{a-b}\right)^{X_{b}} .
\end{aligned}
$$

In the above equations by tending $z$ to $a$ and $b$, we have the next theorem.

Theorem 6. For a linear transform $\sigma$ which preserves $\Sigma \cup\{\infty\}$ and interchanges $a$ and $b$, two points nearest each other in $\Sigma$, we have

$$
\begin{aligned}
\Phi_{\Sigma}^{a b}\left(X_{c} ; c\right. & \in \Sigma) \\
& =\left(\frac{b-\sigma(\infty)}{a-\sigma(\infty)}\right)^{X_{a}}\left(\sum_{W} \mathcal{L}_{\Sigma}^{a b}\left(\operatorname{reg}^{a b} \circ \tau(w)\right) W\right)\left(\frac{b-\sigma(\infty)}{a-\sigma(\infty)}\right)^{X_{b}},
\end{aligned}
$$

where $\tau:=\sigma \circ S$. Equivalently, the inverse of $\Phi_{\Sigma}^{a b}\left(X_{c} ; c \in \Sigma\right)$ can be written as

$$
\Phi_{\Sigma}^{a b}\left(X_{c} ; c \in \Sigma\right)^{-1}=\left(\frac{a-\sigma(\infty)}{b-\sigma(\infty)}\right)^{X_{b}} \Phi_{\Sigma}^{a b}\left(\sigma\left(X_{c}\right) ; c \in \Sigma\right)\left(\frac{a-\sigma(\infty)}{b-\sigma(\infty)}\right)^{X_{a}}
$$


In the special case, for $w \in \mathcal{A}_{\Sigma}^{a b}$ there holds Euler's inversion formula

$$
\sum_{w_{1} w_{2}=w} \operatorname{Li}_{\Sigma}^{a b}\left(\tau\left(w_{1}\right) ; \sigma(z)\right) \operatorname{Li}_{\Sigma}^{a b}\left(w_{2} ; z\right)=\mathcal{L}(w)
$$

and a "duality formula"

$$
\mathcal{L}(w)=\mathcal{L}(\tau(w))
$$

The map $\tau$ is involutive because $\sigma \circ S=S \circ \sigma$ and both $\sigma$ and $S$ are involutive. So it is appropriate to call the equation (26) the "duality formula".

Example 3. Let $\Sigma=\{0,1\}, \sigma(z)=1-z$ and $b=0$ (i.e. $a=1$ ). Then $\tau$ is the anti-involution defined by

$$
x_{0} \mapsto-x_{1}, \quad x_{1} \mapsto-x_{0} .
$$

Define $\mathfrak{h}^{0}=\mathcal{A}_{\Sigma}^{10}, x=x_{0}, y=-x_{1}$, and $\zeta: \mathfrak{h}^{0} \rightarrow \mathbb{R}$ for $w=x^{k_{1}-1} y \cdots x^{k_{r}-1} y \in$ $\mathfrak{h}^{0}$ by

$$
\begin{aligned}
\zeta(w):=\mathcal{L}_{\Sigma}^{a b}(w) & =\sum_{m_{1}>m_{2}>\cdots>m_{r}} \frac{1}{m_{1}^{k_{1}} m_{2}^{k_{2}} \ldots m_{r}^{k_{r}}} \\
& =\zeta\left(k_{1}, k_{2}, \ldots, k_{r}\right) ;
\end{aligned}
$$

then $\tau$ is the anti-involutive mapping

$$
x \mapsto y, \quad y \mapsto x,
$$

satisfying the next relation;

$$
\zeta(\tau(w))=\zeta(w) .
$$

This is the formulation of the duality formula by [7].

The inverse of $\Phi_{\Sigma}^{10}\left(X_{0}, X_{1}\right)$ can be computed as

$$
\begin{aligned}
\Phi_{\Sigma}^{10}\left(X_{0}, X_{1}\right)^{-1} & =\left(\sum_{W} \mathcal{L}_{\Sigma}^{10}\left(\operatorname{reg}^{10} \circ \sigma \circ S(w)\right) W\right)^{-1} \\
& =\sum_{W} \mathcal{L}_{\Sigma}^{10}\left(\operatorname{reg}^{10} \circ \sigma(w)\right) W=\sum_{W} \mathcal{L}_{\Sigma}^{10}\left(\operatorname{reg}^{10}(w)\right) \sigma(W) \\
& =\Phi_{\Sigma}^{10}\left(\sigma\left(X_{0}\right), \sigma\left(X_{1}\right)\right)=\Phi_{\Sigma}^{10}\left(X_{1}, X_{0}\right) .
\end{aligned}
$$

This relation is nothing but (2). 
Example 4. Take $\Sigma=\{0, \pm 1, \pm \sqrt{-1}\}, \sigma=(1-z) /(1+z)$ and $b=0$. Then for any word of $\mathcal{A}_{\Sigma}^{a b}, \mathcal{L}_{\Sigma}^{a b}$ corresponds to the multiple $L$-value of $m=4$. For $w=x_{0}^{k_{1}-1} x_{c_{1}} \cdots x_{0}^{k_{r-1}-1} x_{c_{r-1}} x_{0}^{k_{r}-1} x_{c_{r}}$ the corresponding value is

$$
\begin{aligned}
\mathcal{L}_{\Sigma}^{a b}(w) & =(-1)^{r} \sum_{m_{1}=r}^{\infty} \sum_{m_{1}>\cdots>m_{r-1}>m_{r}>0} \frac{c_{1}^{-m_{1}+m_{2}} \cdots c_{r-1}^{-m_{r-1}+m_{r}} c_{r}^{-m_{r}}}{m_{1}^{k_{1}} \cdots m_{r-1}^{k_{r-1}} m_{r}^{k_{r}}} \\
& =(-1)^{r} L\left(k_{1}, \ldots, k_{r-1}, k_{r} ; a_{1}, \ldots, a_{r-1}, a_{r}\right),
\end{aligned}
$$

where $(\sqrt{-1})^{-a_{i}}=c_{i}$. The involution $\sigma$ interchanges the singular points

$$
\sigma: \quad 0 \longleftrightarrow 1, \quad \sqrt{-1} \longleftrightarrow-\sqrt{-1}, \quad-1 \longleftrightarrow \infty,
$$

so the action of $\sigma$ on $\mathcal{A}_{\Sigma}$, the action of $\sigma$ on $\mathbb{C}\left\langle\left\langle X_{c} ; c \in \Sigma\right\rangle\right\rangle$, and the action of the anti-involution $\tau=\sigma \circ S$ on $\mathcal{A}_{\Sigma}$ are as follows:

$$
\begin{aligned}
& \sigma: \quad x_{0} \mapsto x_{1}-x_{-1}, \quad x_{1} \mapsto x_{0}-x_{-1}, \quad x_{-1} \mapsto-x_{-1}, \\
& x_{\sqrt{-1}} \mapsto x_{-\sqrt{-1}}-x_{-1}, \quad x_{-\sqrt{-1}} \mapsto x_{\sqrt{-1}}-x_{-1}, \\
& \sigma: \quad X_{1} \mapsto X_{0}, \quad X_{-1} \mapsto-X_{0}-X_{1}-X_{\sqrt{-1}}-X_{-1}-X_{-\sqrt{-1}}, \\
& X_{0} \mapsto X_{1}, \quad x_{\sqrt{-1}} \mapsto X_{-\sqrt{-1}}, \quad X_{-\sqrt{-1}} \mapsto X_{\sqrt{-1}}, \\
& X_{x_{0}} \mapsto-x_{1}+x_{-1}, \quad x_{0}+x_{-1}, \quad x_{-1} \mapsto x_{-1}, \\
& x_{\sqrt{-1}} \mapsto-x_{-\sqrt{-1}}+x_{-1}, \quad x_{-\sqrt{-1}} \mapsto-x_{\sqrt{-1}}+x_{-1} .
\end{aligned}
$$

We have the duality formula of multiple $L$-values

$$
\mathcal{L}_{\Sigma}^{a b}(\tau(w))=\mathcal{L}_{\Sigma}^{a b}(w), \quad w \in \mathcal{A}_{\Sigma}^{a b},
$$

which is equivalent to the formula:

$$
\begin{aligned}
& \Phi_{\Sigma}^{a b}\left(X_{0}, X_{1}, X_{\sqrt{-1}}, X_{-1}, X_{-\sqrt{-1}}\right)^{-1} \\
& =2^{X_{0}} \Phi_{\Sigma}^{a b}\left(\sigma\left(X_{0}\right), \sigma\left(X_{1}\right), \sigma\left(X_{\sqrt{-1}}\right), \sigma\left(X_{-1}\right), \sigma\left(X_{-\sqrt{-1}}\right)\right) 2^{X_{1}} .
\end{aligned}
$$

The transform $\sigma$ also preserves the subset $\{0,1,-1, \infty\} \subset \Sigma \cup\{\infty\}$. So the above duality formula can be restricted from $\Sigma$ to $\Sigma^{\prime}=\{0,1,-1\}$. The duality formula for $\Sigma^{\prime}$ is the reproduction of equation (127) of [3].

\section{Acknowledgements}

The author would like to express his grantitude to Saburo Kakei, Michitomo Nishizawa and Yuji Yamada for useful communications. He also thanks Kimio Ueno for valuable advice and comments. 


\section{References}

[1] T. Arakawa and M. Kaneko. On multiple $L$-values. to appear in J. Math. Soc. Japan, 2002.

[2] J. M. Borwein, D. M. Bradley, D. J. Broadhurst, and P. Lisoněk. Special values of multiple polylogarithms. Trans. Amer. Math. Soc., 353(3):907941, 2001.

[3] D. J. Broadhurst. Massive 3-loop Feynman diagrams reducible to SC* primitives of algebras of the sixth root of unity. Eur. Phys. J. C Part. Fields, 8(2):313-333, 1999.

[4] V. G. Drinfel'd. On quasitriangular quasi-Hopf algebras and on a group that is closely connected with $\operatorname{Gal}(\overline{\mathbb{Q}} / \mathbb{Q})$. Algebra $i$ Analiz, 2(4):149-181, 1990.

[5] H. Furusho. The multiple zeta value algebra and the stable derivation algebra. Publ. Res. Inst. Math. Sci., 39(4):695-720, 2003.

[6] N. M. Hoang, M. Petitot, and J. van der Hoeven. Computation of the monodromy of generalized polylogarithms. In Proceedings of the 1998 International Symposium on Symbolic and Algebraic Computation (Rostock), pages 276-283 (electronic), New York, 1998. ACM.

[7] M. E. Hoffman. The algebra of multiple harmonic series. J. Algebra, 194(2):477-495, 1997.

[8] K. Ihara, M. Kaneko, and D. Zagier. Derivation and double shuffle relations for multiple zeta values. preprint, 2003.

[9] J. Okuda and K. Ueno. Relations for Multiple Zeta Values and Mellin Transforms of Multiple Polylogarithms. to appear in Publications of RIMS, 2003, arXiv:math.NT/0301277.

[10] C. Reutenauer. Free Lie algebras, volume 7 of London Mathematical Society Monographs. New Series. The Clarendon Press Oxford University Press, New York, 1993. Oxford Science Publications.

[11] D. Zagier. Values of zeta functions and their applications. In First European Congress of Mathematics, Vol. II (Paris, 1992), volume 120 of Progr. Math., pages 497-512. Birkhäuser, Basel, 1994. 
Department of Mathematical Sciences, School of Science and Engineering, WASEDA UNIVERSITY, 3-4-1, Okubo Shinjuku-ku, Tokyo 169-8555, Japan

okuda@gm . math . waseda.ac.jp 